\title{
Supplementary Material: Sensitivity Analysis for Bayesian Hierarchical Models
}

\author{
Małgorzata Roos * and Thiago G. Martins ${ }^{\dagger}$ and Leonhard Held ${ }^{\ddagger}$ and Håvard Rue ${ }^{\S}$
}

This Supplement contains additional results obtained for a conjugate example, for time series and the spatial variation in survival data. Only two ingredients are necessary for the $\epsilon$-local sensitivity of the model component of interest $\theta$ to be computed. Firstly, the knowledge about the base prior distribution $\pi_{\gamma_{0}}(\theta)$ and its parameter values $\gamma_{0}$ is needed. Secondly, the corresponding marginal posterior density estimate $\left(\theta^{(j)}, \tilde{\pi}_{\gamma_{0}}\left(\theta^{(j)} \mid \boldsymbol{y}\right)\right)$ provided numerically at a finite discrete set, $j=1, \ldots, J$, is required. In the following examples the marginal posterior density estimates were contributed by R-INLA framework, whereas the ready-to-use priorSens package in $R$ facilitated the epsilon grid search and computation of the $\epsilon$-local sensitivity estimates. The agreement of exact analytical (theoretical) and inla-driven sensitivity estimates reassures that our code is working correctly. Moreover, the results in Tables 1 and 2 show that our sensitivity measure is robust with respect to the choice of the $\epsilon$ value for the grid. Apart of that, the desired influence of the sample size on the sensitivity measure is exemplified. Consideration of miscellaneous complex Bayesian hierarchical models including "iid", "ICAR", "spde", "rw1" and "rw2" latent models on several different data sets leads to even more exciting findings. More super-sensitivities were found in Section 4 for "iid" and "ICAR" latent models. In addition, smoothing of two covariates with "rw1" and "rw2" lead to clearly increased sensitivity estimates. To our surprise we found no overly elevated sensitivity values for "spde" components hyperpriors.

\section{A conjugate example for precision}

Assume that $m=2, n=10, n \hat{\sigma}_{M L}^{2}=\sum_{i=1}^{n}\left(y_{i}-m\right)^{2}=0.35$ for observations from iid $\mathrm{N}\left(m, \kappa^{-1}\right)$ with the value of $m$ being fixed and $\kappa \sim \mathrm{G}(\alpha, \beta)$ leading, by conjugacy, to $\kappa \mid \boldsymbol{y} \sim \mathrm{G}\left(\alpha+n / 2, \beta+\sum_{i=1}^{n}\left(y_{i}-\right.\right.$ $\left.m)^{2} / 2\right)$. Fix the base prior parameter values at $\gamma_{0}=\left(\alpha_{0}, \beta_{0}\right)=(0.5,0.01)$.

For $\epsilon_{0}=0.00354$ we computed circular and worst-case sensitivity for both the analytically known posterior as well as for the marginal posterior density obtained form inla and got identical estimates $\mathrm{S}_{\gamma_{0}}^{\text {exact }}\left(\epsilon_{0}\right)=0.21$ and $S_{\gamma_{0}}^{\text {inla }}\left(\epsilon_{0}\right)=0.21$ (see Figure 1), respectively. In spite of the small sample size the mean change in the unit-variance normal distributions for posteriors was only $21 \%$ of the mean change in the unit-variance normal distributions for priors. We found absolute and relative error ranges of the inla-driven circular sensitivity estimates with respect to the analytical ones equal to (-2.8e-05, $1.1 \mathrm{e}-05)$ and $(-4 \mathrm{e}-04,1 \mathrm{e}-04)$. Sensitivity estimates stayed stable over the wide range of $\epsilon$ values (see Table 1) and reacted correctly to the increased sample size.

\section{Latent models}

In the applications provided in the following sections we use several latent models in R-INLA such as "iid", "ICAR", "rw1", "rw2" and stochastic partial differential equations ("spde"). Here we describe them shortly.

${ }^{*}$ Division of Biostatistics, Institute for Social and Preventive Medicine, University of Zurich, Zurich, Switzerland mroos@ifspm.uzh.ch

'Department of Mathematical Sciences, Norwegian University for Science and Technology, Trondheim, Norway guerrera@math.ntnu.no

+Division of Biostatistics, Institute for Social and Preventive Medicine, University of Zurich, Zurich, Switzerland leonhard.held@ifspm.uzh.ch

3.Department of Mathematical Sciences, Norwegian University for Science and Technology, Trondheim, Norway hrue@math.ntnu.no 


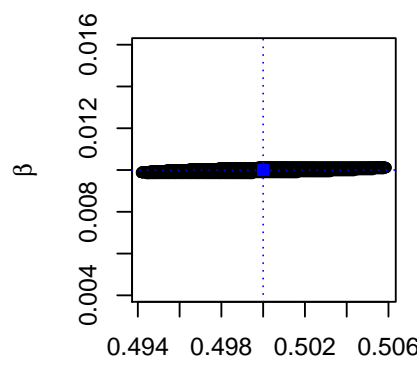

$\alpha$

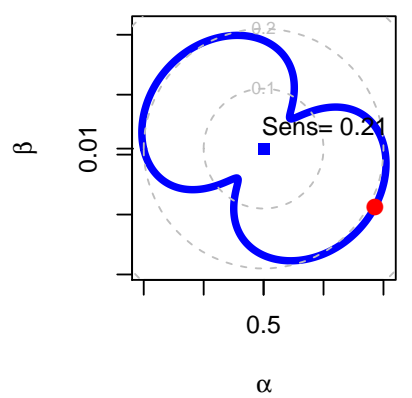

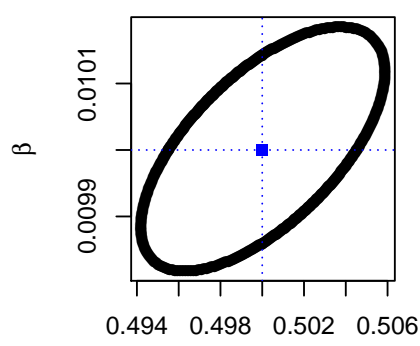

$\alpha$

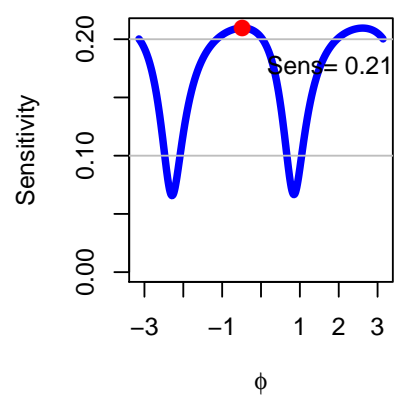

Figure 1: Epsilon grid $\mathrm{G}_{\boldsymbol{\gamma}_{0}}\left(\epsilon_{0}\right)$ with (top left) and without (top right) equal scale on both axes and inla-driven circular $S_{\gamma_{0}}^{c}\left(\epsilon_{0}\right)$ and worst-case sensitivity $S_{\gamma_{0}}\left(\epsilon_{0}\right)$ (red dot) plots for the example in Section 1 at $\gamma_{0}=\left(\alpha_{0}, \beta_{0}\right)=(0.5,0.01)$ and $\epsilon_{0}=0.00354$ : polar sensitivity plot (bottom left) centered at $\gamma_{0}$ and sensitivity plot rolled out on the line (bottom right) for each polar direction. The scale for sensitivity values $(0.1,0.2, \ldots, 0.9,1)$ is indicated by grey circles and lines, respectively.

Table 1: Worst-case sensitivity estimates for precision as a function of $\epsilon$ for $n=10$ and $n=100$ in the example in Section 1 at $\gamma_{0}=\left(\alpha_{0}, \beta_{0}\right)=(0.5,0.01)$.

\begin{tabular}{lcccc}
\hline$\epsilon$ & $\mathrm{S}_{\boldsymbol{\gamma}_{0}}^{\text {exact }}(\epsilon), n=10$ & $\mathrm{~S}_{\boldsymbol{\gamma}_{0}}^{\text {inla }}(\epsilon), n=10$ & $\mathrm{~S}_{\boldsymbol{\gamma}_{0}}^{\text {exact }}(\epsilon), n=100$ & $\mathrm{~S}_{\gamma_{0}}^{\text {inla }}(\epsilon), n=100$ \\
\hline 0.0001 & 0.21 & 0.21 & 0.07 & 0.07 \\
0.0005 & 0.21 & 0.21 & 0.07 & 0.07 \\
0.0010 & 0.21 & 0.21 & 0.07 & 0.07 \\
0.0050 & 0.21 & 0.21 & 0.07 & 0.07 \\
0.0100 & 0.21 & 0.21 & 0.07 & 0.07
\end{tabular}


The unstructured independent random noise model ("iid") for random effects in the vector $\boldsymbol{v}$ defines the Gaussian random field to be a vector of independent Gaussian distributed random variables $v_{j} \stackrel{\text { ind }}{\sim} \mathrm{N}\left(0, \tau_{i i d}^{-1}\right)$ with precision $\tau_{i i d}$, for which the gamma prior is assumed. A more involved structured intrinsic conditional autoregressive model ("ICAR" called also "besag") (Besag et al. 1991) in component $\boldsymbol{u}$ assumes that conditions for neighbouring random effects tend to be similar. The Gaussian random field $\boldsymbol{u}=\left(u_{1}, u_{2}, \ldots, u_{n}\right)$ is defined as

$$
u_{i} \mid u_{j}, i \neq j, \tau_{I C A R} \sim \mathrm{N}\left(\frac{1}{n_{i}} \sum_{i \sim j} u_{j}, \frac{1}{n_{i} \tau_{I C A R}}\right),
$$

where $i \sim j$ indicates that two random effects $i$ and $j$ are neighbours and $n_{i}$ is the number of neighbouring entities of the $i$ th object. In order to guarantee the identifiability of the intercept the option constr $=$ TRUE, a sum-to-zero constraint on each connected component, is used. For "ICAR" precision $\tau_{I C A R}$ the gamma prior is assumed. Both "iid" and "ICAR" latent models are used in Section 4.

The first and second-order intrinsic Gaussian Markov random fields (IGMRFs) are frequently used to model smooth, non-linear functions of covariates in one dimesion (Held and Rue 2010). In both models the precision $\tau$ governs the smoothness of the resulting random effect. For a detailed description of the "rw1" latent model see Section 3 . Rue and Held (2005, equation (3.39)) define the joint density of $\boldsymbol{x} \mid \tau$ for the "rw2" model by

$$
\pi(\boldsymbol{x} \mid \tau) \propto \tau^{(n-2) / 2} \exp \left(-\frac{\tau}{2} \boldsymbol{x}^{T} \mathbf{R} \boldsymbol{x}\right)
$$

where the structure matrix $\mathbf{R}$ is determined by the second-order random walk (Rue and Held 2005 , equation (3.40)).

Recently, Lindgren et al. (2011) provided an explicit link between continuously indexed Gaussian fields and discretely indexed Gaussian Markov random fields using an approximate stochastic weak solution to linear stochastic partial differential equations (SPDEs). The approach developed there was successfully applied to global temperature reconstruction and hierarchical spatio-temporal analysis (Cameletti et al. 2012) and has the potential to model not only Matérn but also non-stationary, nonseparable, anisotropic, oscillation, space-time and multivariate fields on manifolds. In principle the SPDE analysis consists of five steps: domain triangulation, definition of the SPDE model object, definition of the inla function, an inla call and extraction of the results for further use and plotting. For implementation of the SPDE approach in inla together with the step-by-step R-code see http:// www.r-inla.org/examples/case-studies and http://www.r-inla.org/examples/tutorials. In Section 4 a "spde" model makes use of the more detailed information contained in the exact point location of the observations. It considers the fine-scale spatial structure of the underlying smooth process (Lindgren 2012, Simpson et al. 2012a|b).

In order to fix the notation we consider covariance function between two individual locations $s_{i}, s_{j}$ of a spatial field $x(\boldsymbol{s})$, of the Matérn form

$$
c_{\nu}\left(s_{i}, s_{j}\right)=\frac{\sigma^{2}}{\Gamma(\nu+d / 2)(4 \pi)^{d / 2} \kappa^{2 \nu} 2^{\nu-1}}\left(\kappa\left\|s_{i}-s_{j}\right\|\right)^{\nu} K_{\nu}\left(\kappa\left\|s_{i}-s_{j}\right\|\right),
$$

where $\nu$ is the Matérn smoothness parameter of the random field, $\kappa$ is a scale parameter, $\sigma^{2}$ is the variance parameter and $K_{\nu}$ is the modified Bessel function of the second kind. The SPDE approach introduced by Lindgren et al. (2011) makes use of the observation that Matérn fields with the covariance function defined above are the stationary solutions to the SPDE

$$
\left(\kappa^{2}-\Delta\right)^{\frac{\alpha}{2}}(\tau x(s))=W(s),
$$

where $\alpha=\nu+d / 2, \Delta=\sum_{j=1}^{d} \frac{\partial^{2}}{\partial s_{j}^{2}}$ is the Laplacian and $W(s)$ is spatial white noise. In this model $\alpha$ controls the smoothness of the realisations. The practical spatial range is governed by $\kappa$ according 
to the approximation suggested by Lindgren et al. $(2011):$ range $\approx \sqrt{8 \nu} / \kappa$. On the other hand the nominal field variance $\sigma^{2}$ is governed by both parameters $\kappa$ and precision $\tau$ due to

$$
\sigma^{2}=\frac{\Gamma(\nu)}{\Gamma(\nu+d / 2)(4 \pi)^{d / 2} \kappa^{2 \nu} \tau^{2}} .
$$

In the acute myeloid leukemia survival data set considered in Section 4 the spatial locations are contained in two dimensions. Therefore, $d=2$ and $s \in \mathbb{R}^{2}$. For analysis of the data Lindgren et al. (2011) assumed an integer $\alpha=2$ implying that the SPDE only involves second order derivatives. This implies that the Matérn smoothness parameter $\nu=\alpha-d / 2=1$. In such a case the field variance can be expressed as $\sigma^{2}=1 / 4 \pi \kappa^{2} \tau^{2}$ and range $\approx \sqrt{8} / \kappa$. Therefore, investigation of the sensitivity of marginal posterior distributions of $\tau$ and $\kappa$ to prior values assumptions is of prime importance as they are an inherent part of nominal range and variance estimates. In order to enhance the readability of the results we denote in the sequel both parameters by $\tau_{S P D E}$ and $\kappa_{S P D E}$ respectively.

\section{Smoothing time series data}

In this application we consider the time series $(n=192)$ on the monthly number of car drivers in Great Britain killed and seriously injured from January 1969 to December 1984 (Harvey and Durbin 1986 : Harvey 1989). Temporal trends in this time series are of interest, since the seat-belt law became effective on 31 January 1983. As in Rue and Held (2005), we apply the square root transformation to the outcome and consider residuals after removal of the seasonal effect for further analysis. Counts observed in the last eight years of the time series $(n=96)$ are taken to study the influence of the sample size (see Figure 2).

Our main goal is the sensitivity estimation of the posterior distribution $\pi(\tau \mid \boldsymbol{y})$ at the base prior parameter specification in a hierarchical model with a latent Gaussian first-order random walk $\boldsymbol{x}$ ("rw1") (Held and Rue 2010), which can be defined as follows: Let $\boldsymbol{x} \mid \tau \sim \mathrm{N}_{n}\left(\mathbf{0},(\tau \mathbf{R})^{-1}\right)$ with the tridiagonal structure matrix $\mathbf{R}$ determined by the first-order random walk (Rue and Held 2005, p. 95) attaining the following values:

$$
\mathbf{R}_{i j}=\left\{\begin{aligned}
1 & \text { if } i=j=1, n \\
2 & \text { if } 1<i=j<n \\
-1 & \text { if } i=j+1, j-1, \\
0 & \text { otherwise }
\end{aligned}\right.
$$

and assume the gamma prior $\mathrm{G}(\alpha, \beta)$ for the hyperparameter $\tau$ :

$$
\pi(\tau)=\frac{\beta^{\alpha}}{\Gamma(\alpha)} \tau^{\alpha-1} \exp (-\beta \tau)
$$

Therefore, by Rue and Held 2005, equation (3.21))

$$
\pi(\boldsymbol{x} \mid \tau)=(2 \pi)^{-(n-1) / 2} \tau^{(n-1) / 2} \exp \left(-\frac{\tau}{2} \boldsymbol{x}^{T} \mathbf{R} \boldsymbol{x}\right) .
$$

Note that rank of $\mathbf{R}$ is $n-1$ and the random walk of first order is an intrinsic GMRF (IGMRF). Assume that $\boldsymbol{y} \mid \boldsymbol{x}, \tau \sim \mathrm{N}_{n}\left(\boldsymbol{x},(\kappa \mathbf{I})^{-1}\right)$ with $\kappa>0$ fixed. Therefore,

$$
\pi(\boldsymbol{y} \mid \boldsymbol{x}, \tau)=(2 \pi)^{-n / 2} \kappa^{n / 2} \exp \left(-\frac{\kappa}{2}(\boldsymbol{y}-\boldsymbol{x})^{T} \mathbf{I}(\boldsymbol{y}-\boldsymbol{x})\right) .
$$

It can be shown that

$$
\pi(\tau \mid \boldsymbol{y})=\frac{1}{\mathrm{C}(\alpha, \beta)} \tau^{\alpha+(n-1) / 2-1}|\mathbf{Q}|^{-1 / 2} \exp \left(-\beta \tau+\frac{1}{2} \boldsymbol{\mu}^{T} \mathbf{Q} \boldsymbol{\mu}\right),
$$



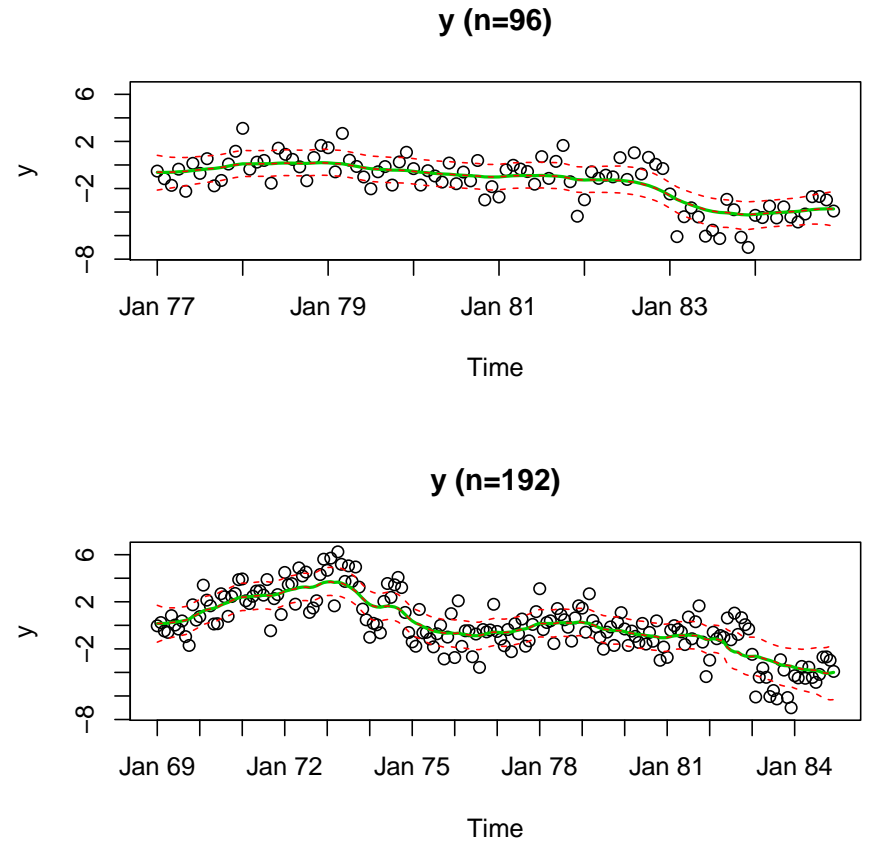

Figure 2: Residuals after removal of a seasonal effect in the square-root-transformed monthly deaths and serious injuries counts in road accidents in Great Britain (Jan1969-Dec1984) together with inla smoothing according to "rw1" (posterior mean together with $0.025,0.5$ and 0.975 quantiles): last eight years of the time series $n=96$ (top), full data $n=192$ (bottom).

where $\mathbf{Q}=\tau \mathbf{R}+\kappa \mathbf{I}, \boldsymbol{\mu}=\mathbf{Q}^{-1} \kappa \boldsymbol{y}^{T}$ and, by an argument provided in the Appendix,

$$
|\mathbf{Q}|=|\tau \mathbf{R}+\kappa \mathbf{I}|=\prod_{i=1}^{n}\left(\tau \lambda_{i}+\kappa\right)=\prod_{i=1}^{n}(\tau(2-2 \cos (\pi(i-1) / n))+\kappa) .
$$

The normalising constant $\mathrm{C}(\alpha, \beta)$ can be computed by numerical integration

$$
\mathrm{C}(\alpha, \beta)=\int_{0}^{\infty} \tau^{\alpha+(n-1) / 2-1}|\mathbf{Q}|^{-1 / 2} \exp \left(-\beta \tau+\frac{1}{2} \boldsymbol{\mu}^{T} \mathbf{Q} \boldsymbol{\mu}\right) d \tau .
$$

The equality

$$
\mathrm{H}\left(\pi\left(\tau \mid \boldsymbol{y}, \alpha_{1}, \beta_{1}\right), \pi\left(\tau \mid \boldsymbol{y}, \alpha_{0}, \beta_{0}\right)\right)=\sqrt{1-\frac{\mathrm{C}\left(\left(\alpha_{0}+\alpha_{1}\right) / 2,\left(\beta_{0}+\beta_{1}\right) / 2\right)}{\sqrt{\mathrm{C}\left(\alpha_{0}, \beta_{0}\right) \mathrm{C}\left(\alpha_{1}, \beta_{1}\right)}}}
$$

enables an analytical estimate of the sensitivity.

In order to guarantee the model conjugacy the precision $\kappa$ was fixed at 0.274 , obtained from the residual variance estimate. We computed both exact and inla-driven sensitivity estimates for $\pi(\tau \mid \boldsymbol{y})$ at the base gamma prior with parameter values $\gamma_{0}=\left(\alpha_{0}, \beta_{0}\right)=(1,0.005)$. They agreed perfectly well giving $\mathrm{S}_{\gamma_{0}}^{\text {exact }}\left(\epsilon_{0}\right)=0.48$ and $\mathrm{S}_{\boldsymbol{\gamma}_{0}}^{\text {inla }}\left(\epsilon_{0}\right)=0.48$ for $\epsilon_{0}=0.00354$, so the mean change in the unit-variance normal distributions for posteriors is $48 \%$ of the mean change in the unit-variance normal distributions for priors. Absolute and relative error ranges of the inla-driven circular sensitivity estimates with respect to the analytical ones were equal to $(-1.5 \mathrm{e}-05,7.1 \mathrm{e}-05)$ and $(-0.000563,0.009528)$, respectively. Moreover, exact and inla-driven estimates were very close for a wide range of $\epsilon$ values and the decreased sample size lead to elevated sensitivity estimates (Table 2). 
Table 2: Worst-case sensitivity estimates for precision $\tau$ as a function of $\epsilon$ for $n=96$ and $n=192$ in Section 3 at $\gamma_{0}=\left(\alpha_{0}, \beta_{0}\right)=(1,0.005)$.

\begin{tabular}{lcccc}
\hline$\epsilon$ & $\mathrm{S}_{\gamma_{0}}^{\text {exact }}(\epsilon), n=96$ & $\mathrm{~S}_{\gamma_{0}}^{\text {inla }}(\epsilon), n=96$ & $\mathrm{~S}_{\gamma_{0}}^{\text {exact }}(\epsilon), n=192$ & $\mathrm{~S}_{\gamma_{0}}^{\text {inla }}(\epsilon), n=192$ \\
\hline 0.0001 & 0.71 & 0.71 & 0.48 & 0.48 \\
0.0005 & 0.71 & 0.71 & 0.48 & 0.48 \\
0.0010 & 0.71 & 0.71 & 0.48 & 0.48 \\
0.0050 & 0.72 & 0.72 & 0.48 & 0.48 \\
0.0100 & 0.73 & 0.73 & 0.49 & 0.49
\end{tabular}

Table 3: Base prior parameter values for the "iid", "ICAR" and "spde" models considered in Section 4

\begin{tabular}{l|l|l|l}
\hline Parameter & "iid" & "ICAR" & "spde" \\
\hline$\beta_{0}, \beta_{1}, \ldots, \beta_{4}$ & $\mathrm{~N}\left(0,0.001^{-1}\right)$ & $\mathrm{N}\left(0,0.001^{-1}\right)$ & $\mathrm{N}\left(0,0.001^{-1}\right)$ \\
$\log (\alpha)$ & $\log \mathrm{G}(0.05,0.1)$ & $\log \mathrm{G}(0.05,0.1)$ & $\log \mathrm{G}(0.05,0.1)$ \\
$\log \left(\tau_{i i d}\right)$ & $\log \mathrm{G}(1,5 \mathrm{e}-5)$ & & \\
$\log \left(\tau_{I C A R}\right)$ & & $\log \mathrm{G}(1,5 \mathrm{e}-5)$ & $\mathrm{N}\left(-3.633,0.1^{-1}\right)$ \\
$\log \left(\tau_{S P D E}\right)$ & & & $\mathrm{N}\left(2.368,0.4^{-1}\right)$ \\
$\log \left(\kappa_{S P D E}\right)$ & & &
\end{tabular}

\section{Spatial variation in survival data}

The analysis of the leukaemia data set from Henderson et al. (2002) leads to a challenging non-conjugate hierarchical model. It encodes spatial variation in 1043 cases of acute myeloid leukaemia (AML) survival in adults diagnosed between 1982 and 1998 in north-west England. Moreover, demographic variables together with the white blood cell count at diagnosis (wbc) and the Townsend score measuring deprivation in the district of residence (tpi) are recorded there. Additionally, spatial information in form of 1043 individual point locations of the AML cases together with their affiliation to one of the 24 administrative districts is provided.

We consider the parametric Weibull proportional hazards model with baseline hazard (Martino et al. 2011)

$$
h(t \mid \boldsymbol{x})=\alpha t^{\alpha-1} \lambda,
$$

where $\alpha$ is the modulus (shape parameter) of the Weibull distribution, a hyperparameter to which a gamma prior distribution is assigned, and the term $\lambda$ is linked to the linear predictor $\boldsymbol{x}^{T} \boldsymbol{\beta}$ in one of the following three ways:

$$
\begin{aligned}
& \log (\lambda)=\boldsymbol{x}^{T} \boldsymbol{\beta}+\boldsymbol{v} \\
& \log (\lambda)=\boldsymbol{x}^{T} \boldsymbol{\beta}+\boldsymbol{u} \\
& \log (\lambda)=\boldsymbol{x}^{T} \boldsymbol{\beta}+\boldsymbol{w}
\end{aligned}
$$

where $\boldsymbol{x}$ is a vector comprising the intercept (const), gender, age, wbc and tpi; $\boldsymbol{\beta}$ is the corresponding vector of the fixed effects parameters; $\boldsymbol{v}$ and $\boldsymbol{u}$ represent the "iid" and "ICAR" models for the districts, respectively, and $\boldsymbol{w}$ denotes the spatial "spde" term for each location.

The simplest district-level "iid" model in $\boldsymbol{v}$ component assumes independence between districts. A more involved district-level "ICAR" model in component $\boldsymbol{u}$ assumes that conditions for AML tend 
Table 4: Worst-case sensitivity estimates for model components in Section 4 for $\epsilon_{0}=0.00354$. Left: wbc and tpi considered directly; Right: models $\left(^{*}\right)$ with rw1 applied to wbc and rw2 to tpi together with discretization in 50 unique equidistant values with default base prior $\mathrm{G}(1,5 \mathrm{e}-5)$.

\begin{tabular}{l|ccc|ccc}
\hline Parameter & "iid" & "ICAR" & "spde" & "iid*" & "ICAR*" & "spde*" \\
& & & & & & \\
\hline const & 0.00469 & 0.00452 & 0.00649 & 0.00534 & 0.00520 & 0.00754 \\
sex & 0.00217 & 0.00216 & 0.00219 & 0.00218 & 0.00218 & 0.00221 \\
age & 0.00007 & 0.00007 & 0.00007 & 0.00007 & 0.00007 & 0.00007 \\
$\operatorname{wbc} / \log \left(\tau_{r w 1(w b c)}\right)$ & 0.00002 & 0.00001 & 0.00002 & 0.83998 & 0.83007 & 0.82776 \\
$\operatorname{tpi} / \log \left(\tau_{r w 2(t p i)}\right)$ & 0.00031 & 0.00030 & 0.00031 & 0.89031 & 0.87505 & 0.88901 \\
$\log (\alpha)$ & 0.00715 & 0.00712 & 0.00750 & 0.00721 & 0.00715 & 0.00757 \\
$\log \left(\tau_{i i d}\right)$ & 1.06591 & & & 0.86448 & & \\
$\log \left(\tau_{I C A R}\right)$ & & 1.12592 & & & 0.94676 & \\
$\log \left(\tau_{S P D E}\right)$ & & & 0.18746 & & & 0.17783 \\
$\log \left(\kappa_{S P D E}\right)$ & & & 0.32776 & & & 0.33474
\end{tabular}

to be similar in neighbouring areas (Henderson et al. 2002, Martino et al. 2011). Political districts are viewed as neighbours if they share a common boundary. The individual-level "spde" model for $\boldsymbol{w}$ makes use of the more detailed information contained in the individual exact point location of the AML patient's residence. Therewith it takes the underlying continuous fine-scale spatial risk process into account (see Section 2 for details).

Our selection of the prior parameter values is based on the choice made by Lindgren et al. (2011) (see Table 3). Base prior parameter values for intercept $\beta_{0}$ and the regression coefficients $\beta_{1}, \ldots, \beta_{4}$ as well as for $\tau_{i i d}$ and $\tau_{I C A R}$ correspond to the default prior parameter values choice assumed by inla. In addition, we assumed independence of priors for log spatial range $\left(\log \left(\kappa_{S P D E}\right)\right)$ and for $\log$ precision $\left(\log \left(\tau_{S P D E}\right)\right)$.

Table 4 (left) shows the inla-driven sensitivity estimates for the three models in Equations (2)(4) with base prior parameter specifications given in Table 3. The hyperparameters of the "iid" and "ICAR" models are super-sensitive. Marginal posterior distributions of the regression coefficients and for Weibull $\alpha$ for all three models do not show much sensitivity at all with the mean change in the unit-variance normal distributions for posteriors being at most $0.7 \%$ of the mean change in the unitvariance normal distributions for priors. Reduced $\tau_{S P D E}$ and $\kappa_{S P D E}$ sensitivity estimates as compared to $\tau_{i i d}$ and $\tau_{I C A R}$ may be implied by a different data use. Whereas both "iid" and "ICAR" models operate on 24 administrative districts, the "spde" geostatistical model accesses the individual location of 1043 AML patients' residence.

Both Kneib and Fahrmeir (2007) and Martino et al. (2011) considered more flexible smooth effects of

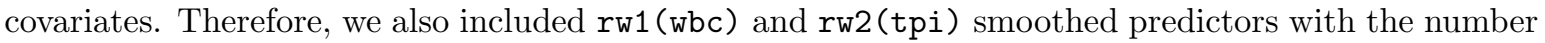
of discretized unique values set to 50 for both variables together with the inla default base prior $\mathrm{G}(1,5 \mathrm{e}-5)$ for the precisions of both latent models. The obtained worst-case sensitivity estimates are reported in Table 4 (right). In general, smoothing of wbc and tpi lead to increased worst-case sensitivity estimates. For example, the mean change in the unit-variance normal distributions for posteriors rw1 (wbc) in the "ICAR" increased to $83 \%$ of the mean change in the unit-variance normal distributions for priors. Interestingly, the super-sensitivity for both "iid" and "ICAR" models disappeared, although their sensitivity values remained high.

The precision for rw1 (wbc) in the "ICAR" model provided us with a typical example for circular 


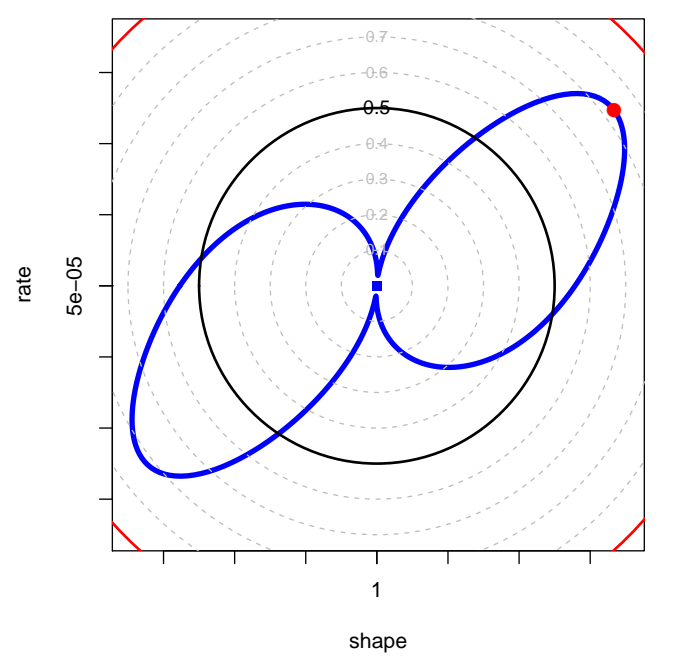

Figure 3: Polar sensitivity plot for the precision of the "rw1" applied to discretized wbc with 50 equidistant values for the "ICAR" model in Section 4 at the default base $\mathrm{G}(1,5 \mathrm{e}-5)$ prior with $\epsilon_{0}=$ 0.00354 . The scale for sensitivity values $(0.1,0.2, \ldots, 0.9,1)$ is indicated by grey circles. Sensitivity equal to 0.5 is marked by a black colour, whereas the sensitivity value 1 is marked by a red one.

sensitivity estimates. Its polar sensitivity values in Figure 3 displaying a diagonal shape indicate that the most pronounced sensitivity modification will be attained if both shape and rate parameters will be changed. Indeed, for example, when shape and rate of the base prior for rw1 (wbc) in the "ICAR" model were set to 0.840 and $4.2 \mathrm{e}-5$ (left lower corner with respect to $(1,5 \mathrm{e}-5)$ ) a lower sensitivity 0.696 was obtained whereas when (shape, rate) values were set to $(1.194,5.9 \mathrm{e}-5)$ (right upper corner with respect to $(1,5 \mathrm{e}-5))$ an increased sensitivity 0.988 was found. For the precision of $\mathrm{rw} 1$ (wbc) median (0.4) and minimal (0.03) sensitivity estimates were calculated.

In contrast, for the precision of $\mathrm{rw2}$ (tpi) a different circular sensitivity plot was generated (Figure 4). The median (0.86) and minimal (0.71) sensitivity estimates were distinct from those obtained for $\mathrm{rw1}_{\mathrm{wbc}} \mathrm{wb}$. Both figures and descriptive sensitivity summaries indicate that although the worstcase sensitivity is comparable, the amount of sensitivity in all directions can considerably differ. We conclude that the precision of $\mathrm{rw2}$ (tpi) is more sensitive at the base prior parameter specification than that for rw1 (wbc).

This example illustrates that assuming the same base prior parameter values $\mathrm{G}(1,5 \mathrm{e}-5)$ for two models, rw1 and rw2, with differing complexity has a distinct meaning. We believe that the promising scaled hyperpriors methodology developed by Sørbye and Rue (2013) and the ideas for penalised complexity priors suggested by Martins et al. (2014) could be further refined by consideration of circular sensitivity estimates.

\section{Appendix}




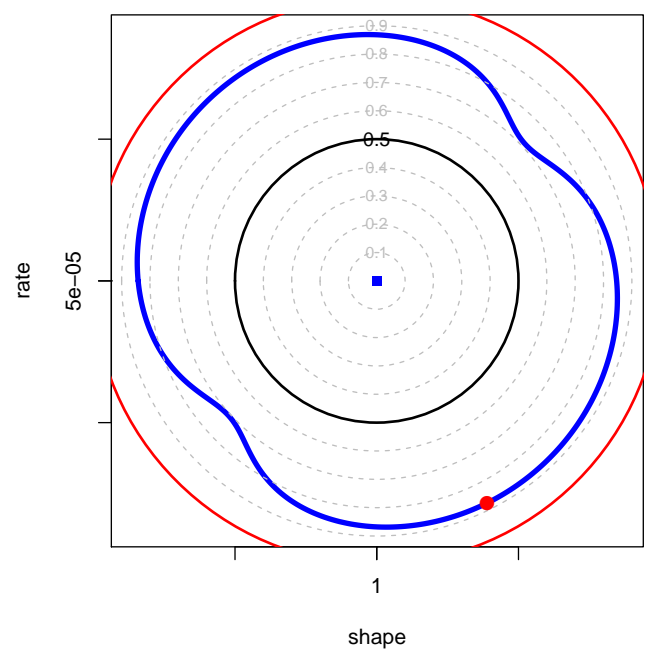

Figure 4: Polar sensitivity plot for the precision of the "rw2" applied to discretized tpi with 50 equidistant values for the "ICAR" model in Section 4 at the default base $\mathrm{G}(1,5 \mathrm{e}-5)$ prior with $\epsilon_{0}=$ 0.00354 . The scale for sensitivity values $(0.1,0.2, \ldots, 0.9,1)$ is indicated by grey circles. Sensitivity equal to 0.5 is marked by a black colour, whereas the sensitivity value 1 is marked by a red one.

Proof of Equation (1): The determinant of the tridiagonal matrix $\mathbf{Q}=\tau \mathbf{R}+\kappa \mathbf{I}$ with values

$$
\mathbf{Q}_{i j}=\left\{\begin{aligned}
\tau+\kappa & \text { if } i=j=1, n \\
2 \tau+\kappa & \text { if } 1<i=j<n \\
-\tau & \text { if } i=j+1, j-1 \\
0 & \text { otherwise. }
\end{aligned}\right.
$$

can be computed explicitly by the following argument:

According to Rue and Held (2005, equation (3.23)) the eigenvalues of the tridiagonal matrix $\mathbf{R}$ are equal to

$$
\lambda_{i}=2-2 \cos (\pi(i-1) / n), i=1, \ldots, n, \text { with } \lambda_{1}=0 .
$$

Note that the eigenvalues of the matrix $\tau \mathbf{R}$ are equal to $\lambda_{i}^{*}=\tau \lambda_{i}$. By Mardia et al. $(1979$, p. 467) the eigenvalues of the matrix $\mathbf{Q}=\tau \mathbf{R}+\kappa \mathbf{I}$ are equal to $\lambda_{i}^{* *}=\tau \lambda_{i}+\kappa$. Therefore, the determinant of the tridiagonal matrix $\mathbf{Q}$ reads

$$
|\mathbf{Q}|=|\tau \mathbf{R}+\kappa \mathbf{I}|=\prod_{i=1}^{n}\left(\tau \lambda_{i}+\kappa\right)=\prod_{i=1}^{n}(\tau(2-2 \cos (\pi(i-1) / n))+\kappa) .
$$

\section{References}

Besag, J., York, J., and Mollié, A. (1991). "Bayesian image restoration, with two applications in spatial statistics." Annals of the Institute of Statistical Mathematics, 43(1): 1-59.

Cameletti, M., Lindgren, F., Simpson, D., and Rue, H. (2012). "Spatio-temporal modeling of particulate matter concentration through the SPDE approach." AStA Advances in Statistical Analysis, 
$1-23$.

URL http://dx.doi .org/10.1007/s10182-012-0196-3

Harvey, A. (1989). Forecasting, Structural Time Series Models and the Kalman Filter. Cambridge: Cambridge University Press.

Harvey, A. and Durbin, J. (1986). "The effects of seat belt legislation on British road causalities: a case study in structural time series modelling." Journal of the Royal Statistical Society, Series A, 149(3): $187-227$.

Held, L. and Rue, H. (2010). "Conditional and intrinsic autoregressions." In Gelfand, A., Diggle, P., Fuentes, M., and Guttorp, P. (eds.), Handbook of Spatial Statistics, 201-216. Chapman \& Hall/CRC.

Henderson, R., Shimakura, S., and Gorst, D. (2002). "Modeling spatial variation in leukemia survival data." Journal of the American Statistical Association, 97(460): 965-972.

Kneib, T. and Fahrmeir, L. (2007). "A mixed model approach for geoadditive hazard regression." Scandinavian Journal of Statistics, 34(1): 207-228.

Lindgren, F. (2012). "Continuous domain spatial models in R-INLA." The ISBA Bulletin, 19(4).

Lindgren, F., Rue, H., and Lindström, J. (2011). "An explicit link between Gaussian fields and Gaussian Markov random fields: the stochastic differential equation approach." Journal of the Royal Statistical Society, Series B., 73(4): 423-498.

Mardia, K. V., Kent, J. T., and Bibby, J. M. (1979). Multivariate Analysis. Academic Press.

Martino, S., Akerkar, R., and Rue, H. (2011). "Approximate Bayesian inference for survival models." Scandinavian Journal of Statistics, 38(3): 514-528.

Martins, T., Simpson, D., Riebler, A., Rue, H., and Sørbye, S. (2014). "Penalising model complexity: A principled, practical approach to constructing priors." (arXiv:1403.4630).

Rue, H. and Held, L. (2005). Gaussian Markov Random Fields. Theory and Applications. Chapman \& Hall/CRC.

Simpson, D., Lindgren, F., and Rue, H. (2012a). "In order to make spatial statistics computationally feasible, we need to forget about the covariance function." Environmetrics, 23(1): 65-74.

- (2012b). "Think continuous: Markovian Gaussian models in spatial statistics." Spatial Statistics, 1: $16-29$.

Sørbye, S. and Rue, H. (2013). "Scaling intrinsic Gaussian Markov random field priors in spatial modelling." Spatial Statistics. 